\title{
Educação em Saúde e suas abordagens integrativas com a Educação Ambiental nas propostas pedagógicas com o uso de filmes: uma análise sobre a produção científica
}

\author{
Educación en Salud y sus abordajes integradores con la Educación \\ Ambiental en las propuestas pedagógicas con el uso de películas: un \\ análisis sobre la producción científica \\ Health Education and its integrative approaches to Environmental \\ Education in pedagogical proposals with the use of films: an analysis of \\ scientific production \\ Aline Teresinha Walczak ${ }^{1}$ \\ Leonardo Priamo Tonello ${ }^{2}$ \\ Eliane Gonçalves dos Santos ${ }^{3}$ \\ Resumo
}

Partindo do entendimento que saúde contempla o conjunto de fatores sociais, ambientais e biológicos, é pertinente que os processos educacionais debatam e apresentem essa compreensão no ensino a fim de que os alunos trabalhem as consequências da ação antrópica no ambiente e na saúde dos sujeitos. Uma maneira de possibilitar estes debates, é a partir do uso de metodologias dinâmicas, tais como os filmes. Este trabalho buscou identificar se são usados filmes comerciais para abordar as questões de saúde correlacionadas a Educação Ambiental. Foi feita uma busca em três repositórios brasileiros. No total de 1153 trabalhos encontrados, cinco atenderam o objetivo da pesquisa. Em suma, há necessidades maiores de estudos e propostas pedagógicas com o uso de filmes na escola que relacione as questões de saúde dentro da perspectiva socioecológica.

Palavras chave: cinema e ensino, metodologias dinâmicas, Saúde e Meio Ambiente

\section{Resumen}

Partiendo del entendimiento que la salud contempla el conjunto de factores sociales, ambientales y biológicos, es pertinente que los procesos educativos debatan y presenten esa comprensión en la enseñanza a fin de que los alumnos trabajen las consecuencias de la acción antrópica en el ambiente y en la salud de los sujetos. Una manera de posibilitar estos debates, es a partir del uso de metodologías dinámicas, tales como las películas. Este trabajo buscó identificar si se usan películas comerciales para abordar las cuestiones de salud relacionadas con la Educación Ambiental. Se realizó una búsqueda en tres repositorios brasileños. En total de 1153 trabajos encontrados, cinco atendieron el objetivo de la investigación. En resumen, hay necesidades mayores de estudios y propuestas pedagógicas con el uso de películas en la escuela que relacione las cuestiones de salud dentro de la perspectiva socioeconómica.

Palabras-clave: cine y enseñanza, metodologías dinámicas, salud y medio ambiente

\begin{abstract}
Based on the understanding that health contemplates a set of social, environmental and biological factors, it is pertinent that educational processes debate and present such understanding in teaching in order to make the

\footnotetext{
${ }^{1}$ Acadêmica de Licenciatura em Ciências Biológicas; Universidade Federal do Rio Grande do Sul - UFRGS, Porto Alegre, Rio Grande do Sul, Brasil; linewalczak@gmail.com Largo, Rio Grande do Sul, Brasil; leonardo.priamo.tonello@gmail.com

${ }^{3}$ Doutora do Programa de Pós-Graduação em Educação nas Ciências; Universidade na Regional do Noroeste do Estado do Rio Grande do Sul - UNIJUÍ. Ijui, Rio Grande do Sul, Brasil; elianesan@bol.com.br
}

${ }^{2}$ Graduando em Ciências Biológicas - Licenciatura; Universidade Federal da Fronteira Sul -UFFS, Cerro
\end{abstract}


students work the consequences of anthropic action in the environment and in the health of the subjects. One way to make these debates possible is by using dynamic methodologies such as films. This work intended to identify if commercial films are used to approach health issues related to Environmental Education. A search was made in three Brazilian repositories. From 1153 papers found, five of them met the objectives of the research. In short, there are greater needs for studies and pedagogical proposals with the use of films in schools that relates the health issues within the socio-ecological perspective.

Keywords: cinema and teaching, dynamic methodologies, Health and Environment

\section{Introdução}

A exploração dos recursos ambientais é um problema social vigente em nosso contexto, decorrente da ação do homem no meio ambiente desde os primórdios, o qual atinge de forma expressiva diversas espécies e gerações que habitam o planeta (LAMBIN et al. 2001; TILMAN et al. 2001; FOLEY et al. 2005; BUTCHART et al. 2010; RANDS et al. 2010), e as consequências desta exploração desenfreada são: o desmatamento, a aceleração no processo de aquecimento global, a poluição de rios, lagos e oceanos, a escassez de água, extinção de espécies, bem como o aumento da população e a ocupação de áreas naturais, que ocasiona urbanização desestruturada, e por consequência a falta de saneamento básico, o aumento de doenças, a exclusão social, assim como o acesso à educação e saúde de qualidade, entre outra questões que atingem de forma mais direta e imediata o homem, e mais especificamente, sua saúde (SCHMIDT, 2007).

Neste sentido, apontamos para a necessidade de ser trabalhado nas escolas as questões pertinentes as problemáticas ambientais e sua correlação com a saúde dos sujeitos de forma transversal e dinâmica, contextualizando e abordando estas questões conforme o contexto social dos sujeitos. Levando em conta que os conhecimentos e visões pertinentes ao meio ambiente e a saúde foram se alterando conforme os contextos históricos e sociais (MARTINS; SANTOS; EL-HANI, 2012). Desta maneira, destacamos a importância de ser utilizadas metodologias didáticas que permitem fazer esta correlação entre os conhecimentos da temática estudada (saúde e meio ambiente) com o período histórico em questão, e é nesse sentido que os filmes se configuram como uma ferramenta de ensino que tem o potencial de atender a esta necessidade.

No momento tecnológico que vivemos, oportunizar debates, reflexões a partir de filmes no ensino é uma oportunidade de instigar e provocar nos alunos uma melhor compreensão das problemáticas que afetam a sociedade do século XXI, pois esse recurso apresenta em seu enredo conhecimentos diversos. Dentro deste pressuposto, destacamos a relevância do ensino de Ciências assumir conformidades didáticas mais dinâmicas e 
contextualizadas, de modo que os alunos se sintam motivados a aprender, a investigar e problematizar, principalmente no que tange aos problemas sociais, como as questões de saúde e ambientais. Neste aspecto, é importante e indispensável que o contexto escolar se intere e se modifique de forma simultânea com o contexto social, para assim se (re)contextualizar e se reformular dentro dos processos de ensino conforme a realidade social dos sujeitos de forma inovadora e interdisciplinar.

Compreendendo estas necessidades e a importância dos processos educacionais se tornem mais atrativos, instigantes e significativos aos alunos (MODRO, 2005), destacamos os filmes comerciais como um recurso didático com o potencial para o ensino, na qual os professores tem a oportunidade de utilizar a tecnologia como uma ferramenta a seu favor, sendo que a utilização dos filmes vem por um viés de facilitar a aprendizagem de conteúdos e a compreensão de conceitos considerados mais abstratos aos alunos, pois diante do enredo do filme, torna-se possível também a construção de aulas mais reflexivas e dialogadas entre aluno e professor, mediante as análises e discussões sobre o tema estudado, abordando as diferentes interfaces da temática em relação aos aspectos: social, ético e científico (SANTOS; PASINI; ANJOS, 2016). Mas, é cabe ressaltar que o planejamento e a mediação pelo professor é essencial para que a aula com filmes resulte em processos de aprendizagem dos alunos.

É importante assinalar que os filmes comercias se configuram como uma metodologia que pode ser utilizada em diversas temáticas trabalhadas ao longo dos diferentes componentes curriculares, dentre eles a Educação em Saúde (ES) co-relacionada com a Educação Ambiental (EA), a partir do viés de que a mesma precisa ser trabalhada e discutida de acordo com um determinado tempo e lugar, ou seja, conforme determinado contexto social, cultural, política e histórico, sendo assim, os filmes permitem a realização destas associações, pois como mencionado anteriormente, a utilização destes possibilita fazer a inserção da temática em questão dentro de diferentes contextos e perspectivas.

Compreendendo saúde como a relação entre o conjunto de fatores sociais, ambientais e biológicos, como as condições de habitação e trabalho, o acesso ao saneamento básico, aos atendimentos de saúde adequados, o acesso à educação, a alimentação e a uma nutrição adequada, assim como um ambiente físico limpo, entre outros (MARTINS; SANTOS; ELHANI, 2012), destacamos as questões de saúde e ambiente como importantes temáticas a serem trabalhadas de maneira interligadas nas escolas, pois, para Mohr (2002) as ações desenvolvidas nas escolas pertinentes a educação em saúde, enfatizando a ação antrópica e o meio ambiente, são fundamentais para a realização da formação integral do sujeito, pois, 
segundo a autora, os comportamentos sociais considerados rotineiros acerca da saúde não garantem que ela ocorra de fato ao máximo na sociedade, sendo considerados apenas informacionistas.

Sob esse ponto, destacamos que a mesma precisa provocar nos alunos o entendimento de que a sua saúde e de sua comunidade está relacionada há vários fatores presentes em seu contexto de vida, principalmente com meio ambiente, para assim refletir e discutir com os alunos a importância de se constituírem cidadãos críticos e participativos perante os problemas sociais, entendendo assim, seu papel como agente na promoção de sua saúde individual e coletiva.

Considerando o exposto até o momento e percebendo a importância da utilização de filmes comerciais como uma ferramenta interdisciplinar, que possibilita a troca de saberes dentro do espaço escolar, bem como a importância de trabalhar questões envolvendo a saúde e a educação ambiental no ensino, o presente trabalho faz referência as análises dos anais de três diferentes eventos, Encontro Nacional de Ensino de Biologia - ENEBIO (2016); VIII Encontro Regional Sul de Ensino de Biologia - EREBIOSUL (2016) e IV Encontro Nacional de Ensino de Ciências da Saúde e do Ambiente ENECiências, de grande relevância para os pesquisadores da área de educação em Ciências, tendo como objetivo traçar um panorama em relação as produções acadêmicas que abordam a utilização de filmes comerciais no ensino para trabalhar questões envolvendo a ES e a EA.

\section{Metodologia:}

O presente trabalho se caracteriza como uma pesquisa qualitativa em educação, conforme os subsídios teóricos de Lüdke e André (2001), a partir da Análise de Conteúdo proposta por Bardin (2009), que se dispõe em três etapas, sendo estas: 1- A pré-análise; 2- A exploração do material; e, por fim, 3 - O tratamento dos resultados: a inferência e a interpretação. A pesquisa foi realizada a partir da análise de trabalhos acadêmicos disponíveis nos anais de três diferentes eventos da área do Ensino de Ciências, a saber: Encontro Nacional de Ensino de Biologia - ENEBIO (2016) e VIII Encontro Regional Sul de Ensino de Biologia - EREBIOSUL (2016), onde os arquivos foram buscados na $9^{\circ}$ Edição da Revista de Ensino de Biologia - RenBio; e do I, II, III e IV Encontro Nacional de Ensino de Ciências da Saúde e do Ambiente ENECiências (2008, 2010, 2012 e 2014), identificando e analisando nos mesmos as publicações referentes ao uso de filmes comercias no ensino, sendo que o número 
total de trabalhos encontrados nos três eventos é de 1153, depois de serem concluídas todas as etapas da Análise de Conteúdo, tem-se como resultado um total de 5 trabalhos.

Após a definição de quais eventos seriam analisados, seguimos com a pesquisa de acordo com as etapas da Análise de Conteúdo da seguinte forma: na Pré-análise, realizamos a busca por documentos nos anais dos respectivos eventos, fazendo a leitura dos títulos, resumos para verificar se estes correspondiam com o objetivo do estudo, que era de encontrar trabalhos que discutem o uso de filmes o ensino de saúde e da educação ambiental como uma ferramenta didática no ensino; na Exploração do Material, realizamos a leitura de todos os documentos selecionados, para assim poder traçar um panorama sobre a utilização de filmes para trabalhar as questões de saúde e ambiente, fazendo posteriormente a categorização de cada documento. Após a análise do material, fizemos o tratamento dos dados, no qual realizamos a sistematização e interpretação dos resultados provenientes da análise e categorização dos documentos.

Os resultados provenientes da análise foram registrados em forma de quadro (Quadro 1), sendo que as publicações selecionadas contém as seguintes informações: 1-Trabalho e autores; 2-Ano de publicação; 3- Nível de ensino, categorizado em i) ensino inicial; ii) ensino fundamental; iii) ensino médio e iv) ensino superior; e 4- tipo de abordagem de Educação em saúde utilizada.

Para análise dos dados, os trabalhos foram nomeados em "T1, T2, ...Tn" sucessivamente, seguindo do número correspondente à ordem cronológica de publicação, sendo que o quadro 1 apresenta uma sistematização das informações coletadas por meio da análise de conteúdo realizada nos trabalhos dos anais dos respectivos eventos e repositório da RenBio.

Quadro 1 - Trabalhos analisados que abordam a utilização de filmes para trabalhar as questões de saúde e

\begin{tabular}{|c|c|c|c|c|c|}
\hline Trabalhos & Titulo & Autor & Ano & Nível de Ensino & $\begin{array}{c}\text { Tipo de } \\
\text { abordagem } \\
\text { utilizada }\end{array}$ \\
\hline $\mathrm{T} 1$ & $\begin{array}{l}\text { Cinema de ficção } \\
\text { científica: } \\
\text { produzindo } \\
\text { conexões } \\
\text { ciências }\end{array}$ & $\begin{array}{lrr}\text { Cilmar } & \text { Castro; } \\
\text { Lucia } & \text { de } & \text { La } \\
\text { Rocque } & & \end{array}$ & 2012 & Ensino superior & Socioecológica \\
\hline $\mathrm{T} 2$ & $\begin{array}{lr}\text { Uma proposta } & \text { de } \\
\text { ensino sobre } & \text { o } \\
\text { Reino Fungi para o } \\
\text { sétimo ano do } \\
\text { ensino } & \\
\text { fundamental, na } \\
\text { perspectiva } & \text { da } \\
\text { teoria } & \text { da } \\
\text { aprendizagem } & \\
\text { significativa } & \end{array}$ & $\begin{array}{l}\text { Luciana Abrão } \\
\text { Lougon Soares; } \\
\text { Evelyse dos Santos } \\
\text { Lemos. }\end{array}$ & 2014 & $\begin{array}{l}\text { Ensino } \\
\text { Fundamental }\end{array}$ & Socioecológica \\
\hline
\end{tabular}


RELACult - Revista Latino-Americana de Estudos em Cultura e Sociedade

\begin{tabular}{|c|c|c|c|c|c|}
\hline $\mathrm{T} 3$ & $\begin{array}{l}\text { A velhice no } \\
\text { século XXI e o } \\
\text { cinema- relações } \\
\text { com o ensino de } \\
\text { biologia }\end{array}$ & $\begin{array}{l}\text { Eliane G. dos } \\
\text { Santos e Maria C. } \\
\text { P. de Araújo }\end{array}$ & 2016 & $\begin{array}{l}\text { Ensino } \\
\text { Fundamental }\end{array}$ & Socioecológica \\
\hline $\mathrm{T} 4$ & $\begin{array}{l}\text { O uso de filmes } \\
\text { comerciais } \\
\text { trabalho } \\
\text { pedagógico } \\
\text { professor: } \\
\text { educação em saúde } \\
\text { em foco }\end{array}$ & $\begin{array}{l}\text { Bruna } \\
\text { Schweinberger e } \\
\text { Eliane G. dos } \\
\text { Santos }\end{array}$ & 2016 & $\begin{array}{l}\text { Ensino } \\
\text { Fundamental e } \\
\text { Ensino Médio }\end{array}$ & Socioecológica \\
\hline T5 & \begin{tabular}{lr}
\multicolumn{2}{l}{ Possíveis } \\
abordagens \\
saúde nos enredos \\
de & filmes \\
comerciais &
\end{tabular} & $\begin{array}{llr}\text { Karine } & \text { Rudek } & \text { e } \\
\text { Eliane } & \text { G. dos } \\
\text { Santos } & & \end{array}$ & 2016 & $\begin{array}{l}\text { Ensino } \\
\text { Fundamental e } \\
\text { Ensino Médio }\end{array}$ & Socioecológica \\
\hline
\end{tabular}

Fonte: AUTORES, 2018.

\section{Resultados e discussão}

Podemos considerar a Educação em Saúde uma temática já tradicional dentro do ensino, pois a mesma pode ser notada nos espaços escolares desde o final do século XIX, consolidando-se principalmente na primeira metade do século XX (COLLARES; MAYSÉS,1985). Contudo, apenas em 1971, por meio da Lei no 5.692 que ela foi formalmente introduzida nos currículos escolares brasileiros. Ainda, é importante ressaltar que assim como nossa sociedade, os processos que envolvem a saúde estão em constante evolução, como por exemplo, a percepção do que é saúde e consequentemente a ES dentro das escolas, na qual vem aumentando a compreensão de que a saúde está diretamente interligada com o meio em que os sujeitos estão inseridos, sendo que é importante enfatizar que o conceito de saúde sempre esteve atrelado ao contexto histórico, social e ambiental dos sujeitos.

Conforme Schmidt (2007) as questões de saúde estão simultaneamente relacionadas com o meio ambiente, e atrelado a isto, a ação antrópica no mesmo, como por exemplo, o crescente aumento do número populacional, a urbanização desordenada, a falta de saneamento básico, a educação, as redes públicas de saúde, construção e ocupação de áreas naturais, desemprego, exclusão social, entre outros fatores. Ainda segundo o autor “(..) Trata-se da ação antrópica desordenada, que neste caso se refere à simples conceituação da ação do homem, desestruturando o ecossistema e viabilizando a aproximação do próprio homem com agentes patógenos desconhecidos.” (2007.p. 375)

De acordo com essas considerações, é possível identificar atualmente três diferentes concepções sobre o que é saúde, e interligado a estas, três diferentes abordagens do tema no ensino: a biomédica, a comportamental e a socioecológica. No modelo biomédico, a saúde é 
caracterizada como a ausência de doença, ou seja, a saúde é vista como a oposição a doença. No modelo comportamental, a saúde é determinada pelas ações individuais do sujeito e familiares que resultam ou não na sua saúde, através das chamadas "mudanças comportamentais" do sujeito com relação aos fatores de risco, não levando em conta fatores que não podem ser gerenciados por eles. Por fim, no modelo socioecológico, a saúde é vista como a interação dos diversos fatores ambientais, sociais e psicológicos do sujeito, ou seja, a saúde está fortemente ligada às reações dos sujeitos frente ao ambiente que o cerca (MARTINS; SANTOS, EL-HANI, 2012).

Desta forma, conforme o quadro 1, foram identificados um total 5 trabalhos que fazem referência a utilização de filmes para trabalhar as questões de saúde pelo viés ambiental, nos diferentes níveis de ensino: Ensino Fundamental (2:5), Ensino Superior (1:5) e Ensinos Fundamental e Médio (2:5). Ainda, o quadro nos permite observar que todos os trabalhos traziam em seus contextos a abordagem socioecológica (5:5), o que consideramos positivo, visto que considerar os fatores ambientais, sociais, culturais e psicológicos, é também considerar a realidade do sujeito, seus contextos, suas práticas, suas observações e interações com o meio. Neste aspecto, é importante enfatizar que não estamos desconsiderando a relevância das abordagens biomédicas e comportamentais, no entanto, apontamos que essas são insuficientes para a construção de uma visão mais ampla do que realmente significa saúde e qualidade de vida e a relação destas com o meio ambiente, visto que as mesmas procuram trabalhar apenas informação pertinentes a presença ou ausência de doenças nos sujeitos e as mudanças comportamentais dos mesmos, sem considerar as condições e o contexto ambiental e social em que estes estão inseridos.

Desta forma, em excertos como: “Embora a descrição das cenas simule muito pouco a imponência destes filmes comerciais, foi possível retirar múltiplas informações para discussão de temas biológicos, ambientais, políticos, culturais e sociais em sala de aula [...]” (T5, 2016); “(..) é papel do ensino de ciências prover subsídios para que o aluno desenvolva a capacidade de compreender a relação dos seres vivos entre si e com os elementos não vivos da natureza e, assim entender a dinâmica ambiental e as implicações da dinâmica social humana na mesma." (T3, 2016) [grifos nossos], podemos perceber como é enfatizado pelos autores dos documentos analisados a importância da abordagem socioecológica no ensino, pois esta permite que haja a formação dos sujeitos por meio da percepção do pertencimento com o meio natural, e que por consequência, todas as ações efetivadas neste tem influência direta na saúde dos mesmos.

Além disso, podemos dizer que por meio da utilização de filmes com o enfoque a 
visão sociecológica, a Saúde deixa de ser um campo de conhecimento como algo estático e higienista, que se apresenta apenas com a relação à ausência ou presença de doenças, e se configura como o conjunto de fatores sociais, biológicos, químicos, físicos, psicossociais, entre outros fatores e condições ambientais que fazem parte do contexto dos sujeitos, conforme o art. $3^{\circ}$ da Lei ${ }^{\circ} 8.080$, de 19 de setembro de 1990, que enfatiza que a saúde

(...) tem como fatores determinantes e condicionantes, entre outros, a alimentação, a moradia, o saneamento básico, o meio ambiente, o trabalho, a renda, a educação, o transporte, o lazer e o acesso aos bens e serviços essenciais; os níveis de saúde da população expressam a organização social e econômica do País. (BRASIL, 1990)

Consideramos que ao fazer a correlação entre as questões de saúde com o meio ambiente, é fomentado a compreensão de como as duas temáticas estão interligadas, sendo importante enfatizar a necessidade dos sujeitos se perceberem como parte pertencente do meio no qual vivem, assim como entenderem como as condições de saúde dependem deste meio. Sauvé (2005) complementa que os problemas socioambientais atuais requerem uma revisão mais profunda desde sua origem, dentro do pressuposto de que a relação com o homem e o meio ambiente tende a ser mantida ao longo do contexto histórico, sendo necessário ter uma nova perspectiva de pertencimento e integração dos sujeitos para com o meio, para que os mesmos tomem consciência da consequências causados a partir das ações de intervenção no meio ambiente, nos processos ambientais e na intensidade da exploração sobre os recursos naturais. Desta forma, acreditamos que os processos educacionais se constituem como potencializadores na construção destas percepções e reconhecimentos, sendo que estes precisam provocar nos alunos o entendimento de que a sua saúde e a da sua comunidade estão relacionadas aos vários fatores presentes em seu contexto de vida, para assim refletir e discutir com eles a importância de se constituírem cidadãos críticos e participativos perante os problemas sociais, entendendo assim, seu papel como agente na promoção de sua saúde individual e coletiva.

Dessa forma, destacamos a importância de utilizar os filmes como uma ferramenta didática para trabalhar questões de Educação em Saúde sobre uma perspectiva de integração com o meio ambiente, dentro do pressuposto de que os filmes possibilitam a a contextualização, a inclusão, a interdisciplinaridade, além da mediação, significação e integração dos saberes (BASTIANI; ROSA 2012; LEMKE, 2014; AUGUSTINHO, et. al, 2012). Além disso, os filmes preservam uma essência dos costumes, cultura e contexto da época em enfoque, permitindo a relação do aluno sob uma equiparação atual e diagnóstica da saúde e dos fatores ambientais como preponderantes de uma visão global em convergências integrativas e plurais, sendo que neste sentido, Fantini (2006) afirma que o cinema apura e 
qualifica a visão, e é portanto um meio para a formação cultural.

Além disso, ressaltamos que o uso de filmes se apresenta como uma resposta à interdisciplinaridade, podendo observar isto a partir dos excertos: “(...) será projetado trechos do filme Os Smurfs" (Sony Pictures Animation, 2011) que, apesar da proposta lúdica, permite aos alunos observar que os organismos interagem entre si e que possuem um papel imprescindível (...).” (T2, 2014); “(...) uso de cinema de fiç̧ão científica, para a discussão de questões mais amplas, indo além da fronteira disciplinar." (T2, 2012) e "O tratamento de assunto de alta complexidade é favorecido pelas imagens e a narrativa contextualizada no cinema." (T1, 2012).

Contudo, de acordo com a literatura da área sinaliza, a temática deve ser trabalhada de forma dinâmica e interdisciplinar, porém autores (MOHR, 2002; ZANCUL, 2017; SAMPAIO, et. al 2014; MARTINS; SANTOS, EL-HANI, 2012; MONTEIRO; BIZZO, 2015) citam que dentre todas as disciplinas estudadas nas diferentes áreas do conhecimento, as questões de saúde e meio ambiente são mais trabalhadas pelas disciplinas correspondentes a área Ciências da Natureza, mais especificamente, no Componente Curricular de Ciências no Ensino Fundamental e Biologia do Ensino Médio, estando presente também em uma pequena parcela na disciplina de Geografia, porém, não se identifica que haja diálogo entre estas áreas do ensino.

Assim, ressaltamos que além da importância de utilizar os filmes como uma metodologia didática para trabalhar questões de saúde e meio ambiente, pois desta forma podem ser feitas maiores reflexões e reconstruções dos conhecimentos dos sujeitos perante a temática, trabalhando-a de forma contextualizada, dinâmica, crítica e reflexiva, para assim, ser realizada a reconstrução de compreensões e saberes sobre o que é ter saúde e quais são medidas necessárias para fazê-la (BRANCO, 2005), deve-se ainda reconhecer dentro dos processos educacionais a necessidade e relevância da temática ser trabalhada de forma interdisciplinar, articulando desta maneira diferentes áreas do conhecimento ao longo das explanações buscando além de dinamizar o ensino, torná-lo mais significativo aos alunos.

\section{Conclusão}

Cada vez mais pesquisadores da área do ensino direcionam o olhar para os processos educacionais, problematizando-o, questionando-o e debatendo-o, como consequência dos diferentes e inúmeros estudos que investigam e contrapõem os entendimentos de Ciência e do ensino de Ciências que foram construídas historicamente e que ainda prevalecem dentro desta escolas, principalmente a concepção do ensino tradicional, no qual geralmente os conteúdos 
são ministrados na disciplina de forma programática, tendo como principal metodologia as aulas expositivas, nas quais o professor é reconhecido como detentor de toda conhecimento e o aluno mero receptor. Desta forma, é destacado a expressiva necessidade dos processos educacionais se reconfigurarem, buscando por meio de novas ferramentas, como a tecnologia, se constituir mais dinâmico, interdisciplinar, motivador e instigante aos alunos.

É importante observar que as escolas se constituem como instituições sociais marcadas pelos processos de socialização, aprendizados, busca e construção de conhecimento, sendo pertinente assim haver nestes espaços o debate sobre as questões ambientais, dentro do pressuposto de que assim os sujeitos, por meio da mediação do professores, podem problematizar e compreender de forma mais significa as relações do homem com a natureza, refletindo, pesquisando, compartilhando experiências e desenvolvendo assim práticas que tenham como objetivo a conservação e cuidado com o meio, desatacando ainda a importância da temática ser trabalha da forma interdisciplinar e dinâmica, buscando estabelecer a partir disso a relação entre o meio com a saúde dos sujeitos, trabalhando como o ambiente influência nas questões de saúde, problematizando e discutindo ainda quais ações que podem ser desenvolvidas com o objetivo de minimizar os danos ambientais e consequentemente, contribuir para a melhoria da saúde de todos os seres vivos.

É importante considerar que na pesquisa documental realizada, observamos a presença do modelo socioecológico em todos trabalhos analisados, fato que consideramos importante, pois esta compreensão permite aos sujeitos a visão da relação da saúde com o ambiente, e do entendimento do sujeito em sua integralidade como o ambiente que o cerca. Entendimento que pode contribuir com ações de preservação e cuidados para com o planeta.

Além disso, por meio do uso das ferramentais midiáticas e da correta mediação do professor, tem-se a oportunidade de potencializar as compreensões sobre saúde voltadas para o indivíduo e sua coletividade, (re)construindo os entendimentos de saúde mais autônomo, no qual o processo de ensino não se limita a uma abordagem de saúde acerca das doenças, do agente causador e da cura, mas sim na troca de saberes e experiências, objetivando ampliar nos sujeitos a visão de saúde, principalmente para o que diz respeito a capacidade dos mesmos relacionar como as questões de saúde estão associadas as condições ambientais, despertando-os para o olhar para as práticas ambientais além do contexto social, político, cultural como determinantes sobre o processo de saúde.

\section{Referencias}


AUGUSTINHO, Elizabeth, VIANA, Sandra da Silva e RÔÇAS, Giselle. O uso do cinema como ferramenta pedagógica para o ensino de Ciências no curso PROEJA. Rio de Janeiro: IFRJ, 2012.

BARDIN, Laurence. Análise de conteúdos. Lisboa: 1977

SCHMIDT, Rosana Andreatta Carvalho. A Questão Ambiental na Promoção da Saúde: uma Oportunidade de Ação Multiprofissional sobre Doenças Emergentes. PHYSIS: Rev. Saúde Coletiva, Rio de Janeiro, 17(2), p. 373-392, 2007.

BASTIANI, Tânia Mara; ROSA, Marcelo Barcellos da. Ética e Cinema: uma Proposta Interdisciplinar para a Educação Ambiental. Revista Monografias Ambientais, Vol. 9, nº 9, p. 2072-2081, 2012.

BRANCO, Isaura Maria Bata Henriques Peixoto. Prevenção do câncer e educação em saúde: opiniões e perspectivas de enfermagem. Texto \& Contexto Enfermagem. v. 14(2), 246-9, Abr./Jun, 2005.

BRASIL. Presidente da República, Lei nº 8080, de 19 de setembro de 1990. Dispõe sobre as condições para a promoção, proteção e recuperação da saúde, a organização e o funcionamento dos serviços correspondentes e dá outras providências.

BUTCHART, Stuart H.M., WALPOLE M., COLLEN B., et al. 2010. Global Biodiversity: Indicators of Recent Declines. Science, 328: 1164-1168.

CHALUH, Laura Noemi. Filmes na formação de futuros professores: educar o olhar.

Educação em Revista. Faculdade de Educação da Universidade Federal de Minas Gerais, v. 28, n. 2, p. 133-152, 2012. Disponível em: http://hdl.handle.net/11449/28890. Acesso dia: 27 de agosto de 2018.

COLLARES, Cecília Azevedo Lima; MOYSÉS, Maria Aparecida Affonso. A transformação do espaço pedagógico em espaço clínico: a patologização da educação. Séries Ideias - FDE, São Paulo, v. 23, p. 25-35, 1997.

ERE-UQAM, Université du Québec à Montréal - Collectif ERE-Francophonie, 2003.

FANTINI, Alvino; TIRMIZI, Aqeel. Exploring and assessing intercultural competence. Federation EIL: Brattleboro, 2006. Disponíel em: https://digitalcollections.sit.edu/worldlearning_publications/1. Acesso dia 27 de agos. De 2018. 
FOLEY, Jonathan A. et al. . Global consequences of land use. Science, 2005, 309: 570-574.

LAMBIN, Eric F. et al. 2001. The causes of land-use and land-cover change: moving beyond the myths. Global Environmental Change, 11: 261-269.

LEMKE, Jay. Investigar para el futuro de la educación cientifica: nuevas formas de aprender, nuevas formas de vivir. Revista Electrónica de Enseñanza de las Ciencias, v. 24, n.1, p. 5-12, mar. 2006.

LÜDKE, Menga; ANDRÉ, Marli Eliza Dalmazo Afonso de. A. Pesquisa em educação: abordagens qualitativas. São Paulo: Epu, 2011.

MARTINS, Liziane; SANTOS, Girlene Silva dos; EL-HANI, Charbel Niño. Abordagens de Saúde em um Livro didático de Biologia Largamente utilizado no Ensino Médio Brasileiro. Investigações em Ensino de Ciências V17(1), p. 249-283, 2012.

MODRO, Nelcimar Ribeiro. Cineducação: usando o cinema em sala de aula. 2005.

MOHR, Adriana. A natureza da educação em saúde no ensino fundamental e os professores de ciências. Tese (Doutorado) - Centro de Ciências da Educação, Universidade Federal de Santa Catarina, Florianópolis. 2002.

MONTEIRO, Paulo Henrique Nico; BIZZO, Nelio. A saúde na escola: análise dos documentos de referência nos quarenta anos de obrigatoriedade dos programas de saúde, 1971-2011. História, Ciências, Saúde - Manguinhos, Rio de Janeiro, v.22, n.2, abr.-jun. 2015, p.411- 427.

RANDS, Michael R. W. et al.. Biodiversity Conservation: Challenges Beyond 2010. Science, 2010, 329: 1298-1303.

SAMPAIO, Aline Firminio. A Temática Educação em Saúde na Formação de Professores de Ciências Naturais. Dissertação (Mestrado Profissional em Ensino de Ciências). Programa de Pós-Graduação em Ensino de Ciências. Universidade de Brasília, Brasília, Brasil, 2014.

SANTOS, Eliane Gonçalves dos; PASINI, Margiéli; ANJOS, Caroline Santos dos. A Possibilidade do Uso dos Filmes na Prática Docente: Uma Análise nos Eventos ANPED e CIECITEC. Revista REnBio, Maringá, n. 9, Out. 2016. Disponível em: http://www.sbenbio.org.br/wordpress/wp-content/uploads/renbio-9/pdfs/1845.pdf. Acesso: 17 set. 2017. 
SAUVÉ, Lucia. Courants et modèles d'interventions en éducation relative à l'environnement.

Module 5. Programme d'études supérieures - Formation en éducation relative à

l'environnement - Francophonie internationale. Montréal : Les Publications

TILMAN, David. el al. 2001. Forecasting agriculturally driven global environmental change. Science, 292: 281-284.

ZANCUL, Mariana de Senzi; GOMES, Paulo Henrique Mendes. A formação de licenciandos em Ciências Biológicas para trabalhar temas de Educação em Saúde na escola. Ensino, Saúde e Ambiente. v. 4, n.1, , p. 49- 61, 2011. 